\title{
Pulmonary Infection with Mycobacterium fortuitum
}

\section{Saba Arshad, Muhammad Khurram, Najia Mahmood", Nida Anjum, Faramerz Khan and Madeeha Nazar}

MU-II, Holy Family Hospital/Rawalpindi medical University, Rawalpindi, Pakistan

"Corresponding author: Mahmood N, MU-II, Holy Family Hospital/Rawalpindi medical University, Rawalpindi, Pakistan, Tel: +92321 5576817; E-mail: najiamahmood33@gmail.com

Received: June 24, 2019; Accepted: July 08, 2019; Published: July 15, 2019

\begin{abstract}
Tuberculosis is caused by Mycobacterium tuberculosis (MTB) which is one of the mycobacteria. Mycobactria other than MTB are termed as non-tuberculous Mycobacteria (NTM). NTM infections are suspected in appropriate clinical scenario when Zeihl Nelson staining of the body fluid demonstrates mycobacteria but Gene X-pert testing for MTB complex is negative. Cultures and drug sensitivity testing are than helpful in establishing NTM infection diagnosis. Mycobacterium fortuitum is one of the NTM. M. fortuitum can cause skin, lymph nodes, joints and pulmonary infections. Here we describe a case report of female who was treated for possible $M$. Fortuitum infection who was diagnosed in similar way.
\end{abstract}

Keywords: Mycobacterium; Tuberculosis; Cultures

\section{Introduction}

The environment contains hundreds of bacteria belonging to mycobacteria species. A number of these are pathogenic i.e., capable of causing serious diseases and infections among humans and other mammals. The most well-known of these mycobacteria is Mycobacterium tuberculosis (TB). The rest of the other mycobacteria not belonging to the M. tuberculosis complex are classified under a single category termed as Mycobacteria other than TB (MOTT). These are also called nontuberculous Mycobacteria (NTM). MOTT or NTM can cause a vast number of diseases and infections other than tuberculosis.

Based on growth pattern, Runyan classified MOTT into four groups [1,2]. Group 1-3 MOTT grow slowly, while group 4 MOTT grow quickly [3]. Group 4 MOTT can be cultured in one week and are termed as rapidly growing mycobacteria (RGM) [4]. Three most common types of RGM that are clinically important are M. abscessus, M. fortuitum, and M. chelonae

Citation: Arshad S, Khurram M, Mahmood N, et al. Pulmonary Infection with Mycobacterium fortuitum. Clin Case Rep Open Access. 2019;2(3):129.

(C)2019 Yumed Text. 
www.yumedtext.com | July-2019

[5]. M. abscessus is the most important human pathogen of this group of bacteria often found in respiratory specimens, whereas $M$. fortuitum is the most commonly encountered organism in clinical practice usually found in non-respiratory systems [6].

M. fortuitum infection can be acquired from environment as well as hospitals setup. Environmental infection with $M$. fortuitum occurs through water, soil, and dust [7-9]. In hospitals settings, patients with impaired immunity, post-surgery, or patients having undergone an invasive procedure through a contaminated instrument are likely to get $M$. fortuitum infection. M. fortuitum can cause skin, lymph nodes, joints and pulmonary infections. Here we describe a case of MOTT pulmonary infection probably caused by M. fortuitum.

\section{Case Report}

A 55 years old housewife, local resident, was admitted with increasing shortness of breath and fever for last two weeks. Her shortness of breath was present for about two years and had worsened recently. It was initially triggered on severe exertion but for last two weeks it had been occurring on minimum exertion and had led to bed bound status.

Shortness of breath was accompanied by cough and productive sputum. The sputum varied from mucoid to purulent. The amount was about $50 \mathrm{ml}$ per day. Hemoptysis was never noted. There was no accompanying orthopnea, chest tightness or chest pain, wheeze, diurnal variation, or palpitations.

Low grade fever had been documented since last one year. Except for respiratory complaints, there was no localizing features. The fever increased in intensity recently and became high grade in last two weeks, with exacerbation of shortness of breath. Fever was associated with rigors and chills. There was no other premorbid illness and systemic inquiry was unremarkable. Based on evaluation of pulmonary features she was put on standard anti-tuberculous therapy for last 2 months. Her fever however did not improve.

At admission, she was distressed, febrile (100 F), and tachypneic (respiratory rate 24). She was using accessory muscles of respiration. Digital clubbing was noted. On examination of chest, chest movements were diminished in right lower chest. Trachea was central, and apex beat normally positioned.

Vocal fremitus was increased in lower right chest. Percussion note was impaired at right lower chest, otherwise it was resonant. Bronchial breathing with increased vocal resonance and fine to medium crackles were noted in right lower chest. At other chest areas breath sounds were harsh vesicular and fine to medium crackles were auscultated at left lower chest. Rest of systemic examination was unremarkable.

She was admitted with diagnosis of pneumonia, and respiratory failure in background of pulmonary TB. Baseline hematological, biochemical investigations, arterial blood gases (ABGs), and cultures were sought. Treatment with intravenous Moxifloxacin, Salbactum potentiated Cefeperazone, and Hydrocortisone along with inhaled bronchodilators and supplemental oxygen was started. Prophylactic Heparin was administered. At admission and serial hematological, biochemical investigations and ABGs are detailed in TABLE 1. Her serial chest X-ray are given in FIG. 1. 


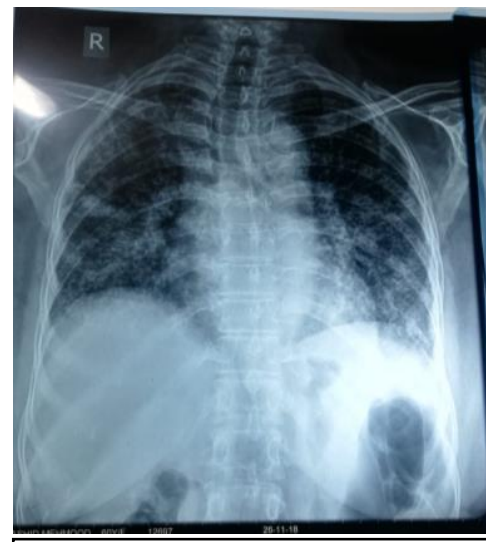

Bilateral diffuse interstitial shadowing seen. Patchy ground glass haze \& shadows seen in right lung field. ILD/Fibrosing alveolitis \& pneumonitis right lung.

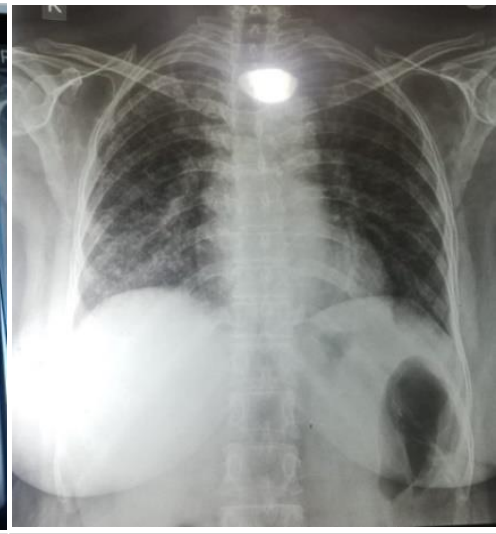

Mild Cardiomegaly. Diffuse airspace infiltrates are seen in bilateral lung field. A welldefined radio opaque opacity is seen in right midzone, could be consolidation.

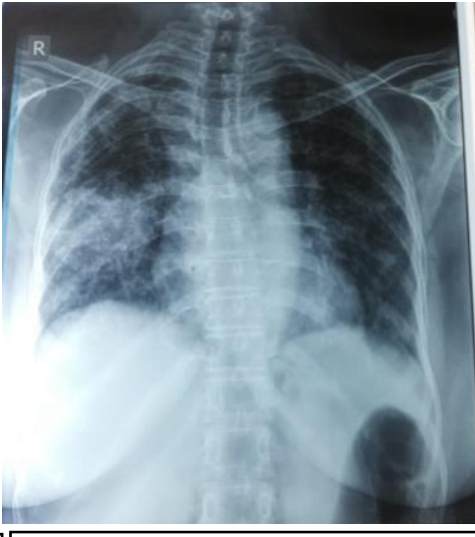

Patchy airspace shadowing throughout both lung fields, suggesting infective etiology.

FIG. 1. Serial Chest X-Rays and Radiologist Reportings.

TABLE 1. Hematological, biochemical investigations and arterial blood gases.

\begin{tabular}{|c|c|c|c|}
\hline Investigations & At admission & $2^{\text {nd }}$ set & At discharge \\
\hline Total leukocyte count & $11300 / \mathrm{mm}^{3}$ & $11100 / \mathrm{mm}^{3}$ & $11000 / \mathrm{mm}^{3}$ \\
\hline Neutrophils & $70 \%$ & $68 \%$ & $65 \%$ \\
\hline Lymphocytes & $21.9 \%$ & $21 \%$ & $20 \%$ \\
\hline Hemoglobin & 10.0 & 10.1 & 10.1 \\
\hline$M C V$ & $96.3 \%$ & $96.2 \%$ & $96.5 \%$ \\
\hline Platelets & 162 & 165 & 170 \\
\hline$E S R$ & 149 & 137 & 103 \\
\hline$A L T$ & 19 & 18 & 19 \\
\hline Bilirubin & 1.1 & 1.1 & 1.0 \\
\hline Alkaline Phosphatase & 166 & 156 & 163 \\
\hline Creatinine & 0.8 & 0.9 & 0.8 \\
\hline Urea & 38 & 39 & 37 \\
\hline Potassium & 3.1 & 3.5 & 3.6 \\
\hline Sodium & 135 & 137 & 138 \\
\hline Chloride & 96 & 98 & 97 \\
\hline$p H$ & 7.527 & 7.489 & 7.4532 \\
\hline $\mathrm{PCO}_{2}$ & $48.6 \mathrm{mmHg}$ & $46 \mathrm{mmHg}$ & $45 \mathrm{mmHg}$ \\
\hline $\mathrm{PO}_{2}$ & $47 \mathrm{mmHg}$ & $69 \mathrm{mmHg}$ & $90 \mathrm{mmHg}$ \\
\hline $\mathrm{SO}_{2}$ & $86.2 \%$ & $93 \%$ & $94 \%$ \\
\hline
\end{tabular}




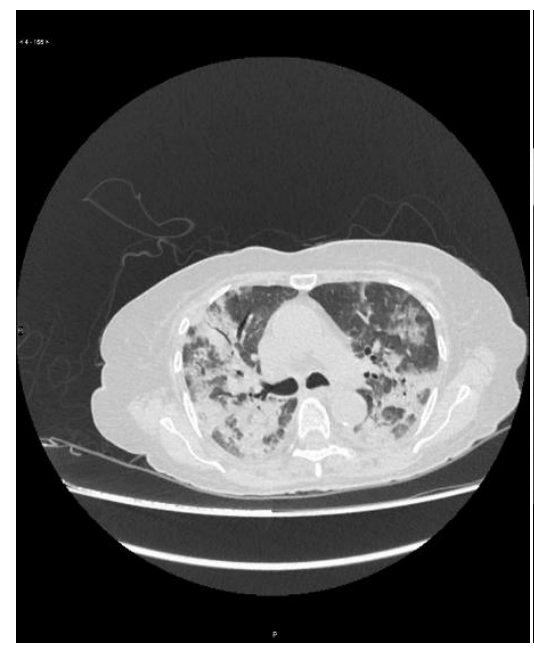

A

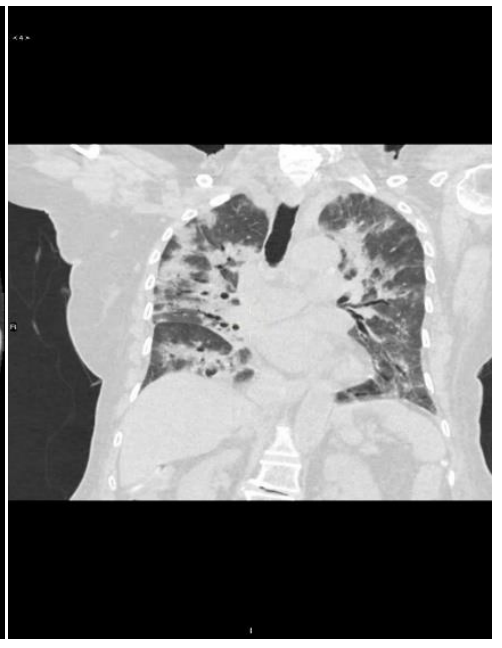

B

FIG. 2. HRCT Chest and Radiologist Reporting.

A \& B. Findings suggestive of hypersensitivity pneumonitis/ extrinsic allergic alveolitis. Interstitial lung disease appears less likely though some basilar interstitial markings are seen. Cardiomegaly and mildly dilated pulmonary trunk.

Patient's condition improved. Her investigations became better (TABLE 1). Sputum microbiological evaluation showed single positive Acid-Fast Bacilli (AFBs). Keeping in mind history of ATT administration, investigations for drug resistance TB were sought. Sputum Gene X-pert for MTB complex turned negative. High resolution Computed Tomography (HRCT) of chest was suggestive of hypersentivity pneumonitis and allergic pneumonitis. Culture of sputum for AFBs was sought, it was positive. AFB culture report showed that the patient was suffering from MOTT infection with likely possibility of M. Fortuitum infection. Results of the AFB sensitivity are given in TABLE 2. Patient was started on culture sensitivity-based treatment for $M$. fortuitum infection. She improved further and was subsequently discharged with advice for regular follow up.

TABLE 2. AFB Antimicrobial Sensitivity Report.

\begin{tabular}{|c|c|}
\hline Medication & AFB sensitivity \\
\hline Amikacin & Sensitive \\
\hline Ciprofloxacin & Sensitive \\
\hline Clarithromycin & Resistant \\
\hline Doxycycline & Resistant \\
\hline Imipenem & Intermediate Sensitive \\
\hline Linezolid & Sensitive \\
\hline Moxifloxacin & Sensitive \\
\hline Trimethoprim/Sulfametxozale & Resistant \\
\hline
\end{tabular}


www.yumedtext.com | July-2019

\section{Discussion}

MOTT infection has been focused in recent studies. A study that included patients from multiple countries pointed rising trend of MOTT infection. According to it, M. fortuitum was most prevalent isolate from Turkey and Iran, and Mycobacterium Avium Complex (MAC) from Europe and South America. In a study from Taiwan increasing trend of RGM infection was noted. MAC infection among RGM was most frequently noted in this study [20].

In a Pakistani study which evaluated multidrug resistant TB amongst TB relapse and treatment failures patients, it was noted that 5\% of mycobacterial isolates were MOTT [21]. Another Pakistani study that analyzed NTM samples from different medical laboratories reported M. xenopi as commonest mycobacteria among MOTT. M. fortuitum was uncommonly noted in this study [22]. In another recent study however higher frequency of RGM (68\%) amongst MOTT was noted. M. fortuitum was commonest among RGM in this study followed by M. mucogenicum and M. smegmatis [23].

A number of human infections can be caused by M. fortuitum irrespective of immune status. M. fortuitum has been isolated from respiratory secretions samples of patients with TB related lung disease, pulmonary malignancy, cystic fibrosis, achalasia, and bronchiectasis etc. Of these, bronchiectasis is most common structural pulmonary disease noted in patients diagnosed to be suffering from $M$. fortuitum pulmonary infection.

It has considered that $M$. fortuitum is a pulmonary colonizer that can cause ephemeral pulmonary infections (transient isolation of $M$. fortuitum during pulmonary pathology evaluation without progression of pulmonary disease and negative successive M is Fortuitum cultures). M. fortuitum however has been noted to cause pulmonary infections in settings of gastrointestinal diseases [10]. Our patient did not have gastrointestinal symptoms. Her CT scan of chest was also not suggestive of findings that predispose to M. fortuitum infection.

Patients with pulmonary RGM infections are typically female, nonsmokers, and post-menopausal [11]. Clinical features of M. fortuitum pulmonary infections include high grade fever, elevation of total leucocyte count and pulmonary infiltrates [12]. In $40 \%$ patients with MOTT pulmonary infection chest X-Ray show either interstitial pattern or mixed interstitial alveolar pattern of shadowing. Reticulonodular pattern is noted in 50 percent of the cases [14]. Our patient had smore or less similar characteristics.

American Thoracic Society (ATS) guidelines are used for diagnosing pulmonary MOTT infections. Clinical signs, radiological features and microbiological evaluations are used for this purpose. At least one of the following microbiological findings must be positive to meet the ATS criteria: 1) positive culture in more than one samples of sputum, 2) positive culture from bronchial wash or lavage, 3) positive culture from a bronchial washing sample with appropriate histopathological findings [13].

Drug Susceptibility Testing (DST) is a crucial criterion for effective management and treatment of patients with MOTT infection. MOTT are not affected by commonly used anti-TB medications [24]. Amikacin, Fluoroquinolones, and Doxycycline are commonly used for treating M. fortuitum infection. M. fortuitum may be sensitive to macrolides on 
www.yumedtext.com | July-2019

sensitivity testing, however macrolides are generally not used due to methylase erm gene induce ability that makes them resistant. Sulfamethoxazole, trimethoprim-sulfamethoxazole, Linezolid, Tigecycline can also be used for M. fortuitum treatment [14].

More than one medication is used to treat M. fortuitum [25]. At least 6 months of treatment is used for M. fortuitum infection. Treatment response is monitored by sputum evaluation after every two months. Medication related side effects/complications should be regularly sought by monitoring renal functions, vestibulocochlear nerve functions, WBC count, and liver functions etc. at 2 monthly intervals.

\section{REFERENCES}

1. Dailloux M, Laurain C, Weber M, et al. Water and nontuberculous mycobacteria. Water Res. 1999;33(10):2219-28.

2. Muthusami JC, Vyas FL, Mukundan U, et al. Mycobacterium fortuitum: an iatrogenic cause of soft tissue infection in surgery. ANZ J Surg. 2004;74(8):662-6.

3. Jarzembowski JA, Young MB. Nontuberculous mycobacterial infections. Arch Pathol Lab Med. 2008;132(8):133341.

4. UpToDate. [cited 23 April 2019]. Available from: https://www.uptodate.com/contents/rapidly-growingmycobacterial-infections-mycobacteria-abscessus-chelonae-and-fortuitum

5. Greenwood D, Barer M, Peutherer J, et al. Medical microbiology. Edinburgh: Churchill Livingstone, UK; 2007.

6. Shitrit D, Baum GL, Priess R, et al. Pulmonary Mycobacterium kansasii infection in Israel, 1999-2004: clinical features, drug susceptibility, and outcome. Chest. 2006;129(3):771-6.

7. Galassi L, Donato R, Tortoli E, et al. Nontuberculous mycobacteria in hospital water systems: application of HPLC for identification of environmental mycobacteria. J Water Health. 2003;1(3):133-9.

8. Petrini B. Non-tuberculous mycobacterial infections. Scand J Infect Dis. 2006;38(4):246-55.

9. Tortoli E. The new mycobacteria: an update. FEMS Immunol Med Microbiol. 2006;48(2):159-78.

10. Okamori S, Asakura T, Nishimura T, et al. Natural history of Mycobacterium fortuitum pulmonary infection presenting with migratory infiltrates: a case report with microbiological analysis. BMC Infect Dis. 2018;18(1):1.

11. Daley C. Rapidly Growing Nontuberculous Mycobacteria (NTM) - Infectious Disease Advisor. Infectious Disease Advisor. 2019 [cited 23 April 2019]. Available from: https://www.infectiousdiseaseadvisor.com/home/decisionsupport-in-medicine/infectious-diseases/rapidly-growing-nontuberculous-mycobacteria-ntm/

12. Park S, Suh GY, Chung MP, et al. Clinical significance of Mycobacterium fortuitum isolated from respiratory specimens. Respir Med. 2008;102(3):437-42.

13. Tanoue L. American Thoracic Society/Centers for Disease Control and Prevention/Infectious Diseases Society of America: Controlling Tuberculosis in the United States. Am J Respir Crit Care Med. 2005;172(9):1169-227.

14. Griffith DE, Girard WM., Wallace RJ Jr. Clinical features of pulmonary disease caused by rapidly growing mycobacteria. An analysis of 154 patients. Am Rev Respir Dis. 1993;147(5):1271-8.

15. Tsukamura M, Shimoide H, Kita N, et al. Epidemiologic studies of lung disease due to mycobacteria other than Mycobacterium tuberculosis in Japan. Rev Infect Dis. 1981;3(5):997-1007.

16. Iseman MD, Marras TK. The importance of nontuberculous mycobacterial lung disease. Am J Respir Crit Care Med. 2008;178(10):999-1000. 
17. Billinger ME, Olivier KN, Viboud C, et al. Nontuberculous mycobacteria-associated lung disease in hospitalized persons, United States, 1998-2005. Emerg Infect Dis. 2009;15(10):1562-9.

18. Thomson RM. Changing epidemiology of pulmonary nontuberculous mycobacteria infections. Emerg Infect Dis. 2010;16(10):1576-83.

19. Kendall BA, Varley CD, Choi D, et al. Distinguishing tuberculosis from nontuberculous mycobacteria lung disease, Oregon, USA. Emerg Infect Dis. 2011;17(3):506-9.

20. Martin-Casabona N, Bahrmand AR, Bennedsen J, et al. Non-tuberculous mycobacteria: patterns of isolation. A multi-country retrospective survey. Int J Tuberc Lung Dis. 2004;13(10):1186-93.

21. Ghafoor A, Mehraj J, Afridi ND, et al. Multidrug resistant Mycobacterium tuberculosis amongst Category I \& II failures and Category II relapse patients from Pakistan. Int J Mycobacteriol. 2012;1(3):118-23.

22. Khanum T, Rasool SA, Ajaz M, et al. Isolation-drug resistance profile and molecular characterization of indigenous typical and atypical mycobacteria. Pak J Pharm Sci. 2011;13(4):527-32.

23. Ahmed I, Jabeen K, Hasan R. Identification of non-tuberculous mycobacteria isolated from clinical specimens at a tertiary care hospital: a cross-sectional study. Available from: https://www.ncbi.nlm.nih.gov/pmc/articles/PMC4016137/\#B14

24. Wallace RJ, Brown BA., Onyi GO. Skin, soft tissue, and bone infections due to Mycobacterium chelonae (chelonae): importance of prior corticosteroid therapy, frequency of disseminated infections, and resistance to oral antimicrobials other than clarithromycin. J Infect Dis. 1992;166(2):405-12.

25. Swenson JM, Wallace RJ, Silcox VA, et al. Antimicrobial susceptibility of five subgroups of Mycobacterium fortuitum and Mycobacterium chelonae. Antimicrob Agents Chemother. 1985;28(6):807-11. 